\title{
THE 2014 ALMA LONG BASELINE CAMPAIGN: FIRST RESULTS FROM HIGH ANGULAR RESOLUTION OBSERVATIONS TOWARD THE HL TAU REGION*
}

\author{
AlMA Partnership, C. L. Brogan ${ }^{1}$, L. M. Pérez ${ }^{2,19}$, T. R. Hunter ${ }^{1}$, W. R. F. Dent ${ }^{3,4}$, A. S. Hales ${ }^{1,3}$, R. E. Hills ${ }^{5}$, \\ S. Corder ${ }^{1,3}$, E. B. Fomalont ${ }^{1,3}$, C. Vlahakis $^{3,4}$, Y. Asaki ${ }^{6,7}$, D. Barkats ${ }^{3,4}$, A. Hirota ${ }^{3,6}$, J. A. Hodge ${ }^{1,19}$,

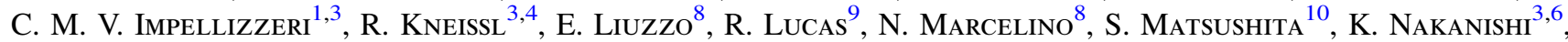
N. Phillips ${ }^{3,4}$, A. M. S. Richards ${ }^{11}$, I. Toledo ${ }^{3}$, R. Aladro ${ }^{4}$, D. Broguiere ${ }^{12}$, J. R. Cortes ${ }^{1,3}$, P. C. Cortes ${ }^{1,3}$, D. Espada ${ }^{3,6}$, F. Galarza ${ }^{3}$, D. Garcia- Appadoo ${ }^{3,4}$, L. Guzman- Ramirez ${ }^{4}$, E. M. Humphreys ${ }^{13}$, T. Jung ${ }^{14}$, S. Kameno ${ }^{3,6}$, R. A. Laing ${ }^{13}$, S. LeON ${ }^{3,4}$, G. Marconi ${ }^{3,4}$, A. Mignano ${ }^{8}$, B. Nikolic ${ }^{5}$, L.-A. Nyman ${ }^{3,4}$, M. Radiszcz ${ }^{3}$, A. Remijan ${ }^{1,3}$, J. A. Rodón ${ }^{4}$, T. Sawada ${ }^{3,6}$, S. Takahashi ${ }^{3,6}$, R. P. J. Tilanus ${ }^{15}$, B. Vila Vilaro ${ }^{3,4}$, L. C. Watson ${ }^{4}$, T. Wiklind ${ }^{3,4}$, E. AkiYama $^{6}$, E. Chapillon $^{12,16,18}$, I. de Gregorio- Monsalvo ${ }^{3,4}$, J. Di Francesco ${ }^{17}$, F. Gueth ${ }^{12}$, A. Kawamura ${ }^{6}$, C.-F. LeE ${ }^{10}$, Q. Nguyen Luong ${ }^{6}$, J. Mangum ${ }^{1}$, V. Pietu ${ }^{12}$, P. Sanhueza ${ }^{6}$, K. Saigo ${ }^{6}$, S. Takakuwa ${ }^{10}$, C. Ubach ${ }^{1}$, T. van Kempen ${ }^{15}$, A. Wootten ${ }^{1}$, A. Castro- Carrizo ${ }^{12}$, H. Francke ${ }^{3}$, J. Gallardo ${ }^{3}$, J. Garcia ${ }^{3}$, S. Gonzalez ${ }^{3}$, T. Hill ${ }^{3,4}$, T. Kaminski ${ }^{4}$, Y. Kurono ${ }^{3,6}$, H.-Y. Liu ${ }^{10}$, C. Lopez ${ }^{3}$, F. Morales ${ }^{3}$, K. Plarre ${ }^{3}$, G. Schieven ${ }^{17}$, L. Testi ${ }^{13}$, L. Videla ${ }^{3}$, E. Villard ${ }^{3,4}$, P. AndREAni ${ }^{13}$, J. E. HibBard ${ }^{1}$, And K. TATEMATSU ${ }^{6}$

${ }^{1}$ National Radio Astronomy Observatory, 520 Edgemont Road, Charlottesville, VA, 22903, USA

${ }^{2}$ National Radio Astronomy Observatory, P.O. Box O, Socorro, NM 87801, USA

${ }^{3}$ Joint ALMA Observatory, Alonso de Córdova 3107, Vitacura, Santiago, Chile

${ }^{4}$ European Southern Observatory, Alonso de Córdova 3107, Vitacura, Santiago, Chile

${ }^{5}$ Astrophysics Group, Cavendish Laboratory, J.J. Thomson Avenue, Cambridge CB3 0HE, UK

${ }^{6}$ National Astronomical Observatory of Japan, 2-21-1 Osawa, Mitaka, Tokyo 181-8588, Japan

${ }^{7}$ Institute of Space and Astronautical Science (ISAS), Japan Aerospace Exploration Agency (JAXA),

3-1-1 Yoshinodai, Chuo-ku, Sagamihara, Kanagawa 252-5210 Japan
${ }^{8}$ INAF, Istituto di Radioastronomia, via P. Gobetti 101, I-40129 Bologna, Italy

${ }^{9}$ Institut de Planétologie et d'Astrophysique de Grenoble (UMR 5274), BP 53, F-38041 Grenoble Cedex 9, France

${ }^{10}$ Institute of Astronomy and Astrophysics, Academia Sinica, P.O. Box 23-141, Taipei 106, Taiwan

${ }^{11}$ Jodrell Bank Centre for Astrophysics, School of Physics and Astronomy, University of Manchester, Oxford Road, Manchester M13 9PL, UK

${ }^{12}$ Institut de Radioastronomie Millimétrique (IRAM), 300 rue de la Piscine, Domaine Universitaire, F-38406 Saint Martin d'Hères, France

${ }^{13}$ European Southern Observatory, Karl-Schwarzschild-Str. 2, D-85748 Garching bei München, Germany

14 Korea Astronomy and Space Science Institute, Daedeokdae-ro 776, Yuseong-gu, Daejeon 305-349, Korea

${ }^{15}$ Leiden Observatory, Leiden University, P.O. Box 9513, 2300 RA Leiden, The Netherlands

${ }^{16}$ Univ. Bordeaux, LAB, UMR 5804, F-33270 Floirac, France

${ }^{17}$ National Research Council Herzberg Astronomy \& Astrophysics, 5071 West Saanich Road, Victoria, BC V9E 2E7, Canada

${ }^{18}$ CNRS, LAB, UMR 5804, 33270 Floirac, France

Received 2015 February 25; accepted 2015 March 17; published 2015 July 14

\section{ABSTRACT}

We present Atacama Large Millimeter/submillimeter Array (ALMA) observations from the 2014 Long Baseline Campaign in dust continuum and spectral line emission from the HL Tau region. The continuum images at wavelengths of $2.9,1.3$, and $0.87 \mathrm{~mm}$ have unprecedented angular resolutions of 0 ". 075 (10 AU) to 0 ". 025 (3.5 AU), revealing an astonishing level of detail in the circumstellar disk surrounding the young solar analog HL Tau, with a pattern of bright and dark rings observed at all wavelengths. By fitting ellipses to the most distinct rings, we measure precise values for the disk inclination $\left(46^{\circ} .72 \pm 0.05\right)$ and position angle $\left(+138^{\circ} .02 \pm 0.07\right)$. We obtain a high-fidelity image of the $1.0 \mathrm{~mm}$ spectral index $(\alpha)$, which ranges from $\alpha \sim 2.0$ in the optically thick central peak and two brightest rings, increasing to $2.3-3.0$ in the dark rings. The dark rings are not devoid of emission, and we estimate a grain emissivity index of 0.8 for the innermost dark ring and lower for subsequent dark rings, consistent with some degree of grain growth and evolution. Additional clues that the rings arise from planet formation include an increase in their central offsets with radius and the presence of numerous orbital resonances. At a resolution of 35 AU, we resolve the molecular component of the disk in $\mathrm{HCO}^{+}(1-0)$ which exhibits a pattern over LSR velocities from 2-12 $\mathrm{km} \mathrm{s}^{-1}$ consistent with Keplerian motion around a $\sim 1.3 M_{\odot}$ star, although complicated by absorption at low blueshifted velocities. We also serendipitously detect and resolve the nearby protostars XZ Tau (A/B) and $\mathrm{LkH} \alpha 358$ at $2.9 \mathrm{~mm}$.

Key words: protoplanetary disks - stars: formation - stars: individual (HL Tau, XZ Tau, LkH $\alpha 358$ ) submillimeter: planetary systems - techniques: interferometric

\footnotetext{
* This article is part of the ALMA Long Baseline Campaign collection. An introduction and the full list of articles can be found at http://iopscience.iop. org/2041-8205/page/Focus_on_the_ALMA_Long_Baseline_Campaign.

19 NRAO Jansky Fellow.
}

\section{INTRODUCTION}

Inside the Taurus star-forming complex, HL Tau is a young star located in a molecular ridge of length $\sim 0.05 \mathrm{pc}$, which forms part of the wall of a large-scale bubble seen in both ${ }^{13} \mathrm{CO}$ 
and faint scattered light (Welch et al. 2000; Anglada et al. 2007). This material contributes to the estimated 24-33 mag of visual extinction $\left(A_{V}\right)$ toward HL Tau (Close et al. 1997; Men'shchikov et al. 1999). Three other less obscured protostars lie toward the edges of this molecular ridge; in order of separation from HL Tau they are XZ Tau, LkH 2358 , and HH30 (e.g., Moriarty-Schieven et al. 2006). Together these four protostars make up the "HL Tau region" in L1551. In this paper we adopt the standard mean distance to Taurus of $140 \mathrm{pc}$ (Rebull et al. 2004) for the HL Tau region. ${ }^{20}$

As a result of the high extinction, the central star of HL Tau has not been directly detected at optical wavelengths, and only a conical reflection nebula has been observed (e.g., Stapelfeldt et al. 1995). Near-infrared (NIR) images reveal a pointlike object that has been attributed to direct stellar radiation, although scattered emission from a central hot disk is also a likely explanation (Close et al. 1997; Men'shchikov et al. 1999). Based on high dispersion optical spectroscopy, HL Tau is classified as spectral type K5 \pm 1 (White \& Hillenbrand 2004). Analyses of the full SED find bolometric luminosities ranging from 3.5 to $15 L_{\odot}$ and classify it as a Class I-II protostar with evidence of an extended envelope and a circumstellar disk (Kenyon \& Hartmann 1995; Men'shchikov et al. 1999; Robitaille et al. 2007). In all, the stellar properties of HL Tau still have a high degree of uncertainty despite extensive study.

Measurements in the millimeter regime suffer far less from extinction effects, and the HL Tau disk is one of the brightest at these wavelengths (Andrews \& Williams 2005); hence HL Tau has been a favored interferometric target over the last two decades (Sargent \& Beckwith 1991; Mundy et al. 1996; Lay et al. 1997; Kitamura et al. 2002; Looney et al. 2000; Guilloteau et al. 2011; Stephens et al. 2014 to name a few). The highest angular resolution millimeter observations to date have a 0 ". 13 (18 AU) beam at $1.3 \mathrm{~mm}$ in which the disk is resolved with an outer radius of $120 \mathrm{AU}$ at a position angle (P.A.) of $+136^{\circ}$ and an inclination (from face-on) of $40^{\circ}$ (Kwon et al. 2011). Modeling of previous millimeter data suggests an HL Tau disk mass of $M_{d} \sim 0.03-0.14 M_{\odot}$ (Robitaille et al. 2007; Guilloteau et al. 2011; Kwon et al. 2011). This high disk mass is within an order of magnitude of previous HL Tau stellar mass estimates ranging from 0.55 to $1.2 M_{\odot}$ (Sargent \& Beckwith 1991; Close et al. 1997; White \& Hillenbrand 2004), suggesting that the disk may be close to being gravitationally unstable.

At longer (centimeter) wavelengths, free-free emission from an outflow jet orthogonal to the disk plane contributes to the continuum (Wilner et al. 1996; Rodmann et al. 2006; Greaves et al. 2008; Carrasco-González et al. 2009). Also known as HH150-151, this jet is seen in optical and NIR shock-tracing lines such as [S II] and [Fe II], extending up to $10,000 \mathrm{AU}$ from the star at velocities of $100-200 \mathrm{~km} \mathrm{~s}^{-1}$ (Mundt et al. 1990; Krist et al. 2008). The inner jet has been traced down to 100 AU from the disk (Pyo et al. 2006; Takami et al. 2007; Beck et al. 2010). At millimeter wavelengths, $\mathrm{CO}$ emission traces a lower-velocity, entrained component of this outflow, mainly showing redshifted gas on the southwest side of the disk (Cabrit et al. 1996; Monin et al. 1996). The central few hundred AU of this outflow was also observed using the SMA in the ${ }^{12} \mathrm{CO}(3-2)$ line (Lumbreras \& Zapata 2014) and showed a similar dominance of redshifted gas. Detailed study of the

\footnotetext{
${ }^{20}$ However, very long baseline interferometry measurements indicate that the line-of-sight depth of the Taurus complex is $\sim 20 \mathrm{pc}$, so that reported linear distances could be in error by up to $15 \%$ (e.g., Loinard 2013).
}

molecular gas associated with the disk has heretofore been plagued by spatial and kinematic confusion due to the extended ridge and outflow emission near the ambient velocity of $\mathrm{v}_{\mathrm{lsr}}=6-7 \mathrm{~km} \mathrm{~s}^{-1}$ (e.g., Cabrit et al. 1996).

Overall, HL Tau is an excellent example of a system just emerging from its protostellar cocoon (i.e., evolving from SED Class I to Class II) that contains a massive compact disk as well as a highly collimated outflow. The system is young ( $\leqslant 1-2 \mathrm{Myr}$ based on the cluster age of Taurus; Briceño et al. 2002), and the high disk mass makes it an ideal candidate for disk instability and early planet formation (e.g., Nero \& Bjorkman 2009). In this paper, we present multi-wavelength Atacama Large Millimeter/ submillimeter Array (ALMA) Science Verification (SV) continuum and spectral line observations from the 2014 Long Baseline Campaign (LBC) of the HL Tau region. These data dramatically demonstrate the revolutionary impact that the full sensitivity and resolution of ALMA will have on the field of star and planet formation. In this work, we aim to give an initial taste of the incredible richness extant in these first ALMA long baseline observations of a protoplanetary disk, and we expect that in time the community will uncover the full scope of what is possible with these data.

\section{OBSERVATIONS AND DATA REDUCTION}

The ALMA SV data on HL Tau were taken between 2014 October 14 and November 14 with baselines ranging from $15 \mathrm{~m}$ to $15.2 \mathrm{~km}$. ALMA Partnership et al. (2015, hereafter ALMA I) gives the detailed tuning frequencies for each band. To summarize, the 1.3 and $0.87 \mathrm{~mm}$ data (Bands 6 and 7) have four $2 \mathrm{GHz}$ (1.875 GHz usable) spectral windows (spws) with 128 channels per spw (hereafter referred to as a "continuum" spw). The $2.9 \mathrm{~mm}$ data (Band 3) were taken with two different tunings. One used three continuum spws, along with two narrow bandwidth spws centered on $\mathrm{HCO}^{+}(1-0)$ and $\mathrm{HCN}(1-0)$, each with a channel resolution of $\sim 0.21 \mathrm{~km} \mathrm{~s}^{-1}$ and a velocity resolution of $0.42 \mathrm{~km} \mathrm{~s}^{-1}$. The other $2.9 \mathrm{~mm}$ tuning used two continuum spws, one narrow bandwidth window on ${ }^{12} \mathrm{CO}(1-0)$, and another spanning four hyperfine transitions of $\mathrm{CN}(1-0)$. These data have a channel resolution of $\sim 0.16 \mathrm{~km} \mathrm{~s}^{-1}$ and a velocity resolution of $0.32 \mathrm{~km} \mathrm{~s}^{-1}$. The scheduling blocks (SBs) were designed to run for 60-70 minutes, with approximately 30 minutes on-source per execution, and each included about 30 antennas. Each of the $2.9 \mathrm{~mm}$ SBs were executed 7 times, while the 1.3 and $0.87 \mathrm{~mm} \mathrm{SBs}$ were executed 9 and 10 times, respectively. The start times were staggered to obtain good hour angle coverage for each band and hence good UV-coverage. It cannot be overstated how crucial the UV-coverage is to the successful imaging of the complex structure of HL Tau.

The calibration and imaging for the continuum and spectral line data are described in the Appendix. The final synthesized beams and rms noise levels for the continuum data are given in Table 1 . Cubes with $0.25 \mathrm{~km} \mathrm{~s}^{-1}$ velocity width channels were made for the four observed lines at $1^{\prime \prime} 1$ angular resolution. Additionally, a $\mathrm{HCO}^{+}(1-0)$ cube with 0 .' 25 resolution was also created (rms noise levels for the cubes are given in the Appendix). All of the images described in this paper are publicly available from the ALMA SV page. ${ }^{21}$

\footnotetext{
21 The raw data, calibration scripts (including detailed absolute flux scale information), and images are available from http://almascience.org/alma-data/ science-verification. Additional details along with the full imaging scripts are available from http://casaguides.nrao.edu/index.php?title=ALMA2014 LBC_SVDATA.
} 
Table 1

Measured Continuum Parameters ${ }^{\mathrm{a}}$

\begin{tabular}{|c|c|c|c|c|c|c|}
\hline \multirow[t]{2}{*}{ Source } & \multicolumn{2}{|c|}{ Position $^{\mathrm{b}}$} & \multirow{2}{*}{$\begin{array}{c}\mathrm{rms} \\
\left(\mu \mathrm{Jy} \mathrm{beam}^{-1}\right)\end{array}$} & \multirow{2}{*}{$\begin{array}{c}\text { Peak }^{\mathrm{c}} \\
\left(\mathrm{mJy} \mathrm{beam}^{-1}\right)\end{array}$} & \multirow{2}{*}{$\begin{array}{l}\text { Flux Density }{ }^{\mathrm{c}} \\
(\mathrm{mJy})\end{array}$} & \multirow{2}{*}{$\begin{array}{c}\text { Size }^{\mathrm{c}} \\
(\operatorname{mas} \times \operatorname{mas}(\operatorname{deg}))\end{array}$} \\
\hline & R.A. (J2000) & Decl. (J2000) & & & & \\
\hline \multicolumn{7}{|c|}{$2.9 \mathrm{~mm}($ Band 3, $101.9 \mathrm{GHz}) 85.3 \times 61.1 \mathrm{mas}$, P.A. $=-179^{\circ}$} \\
\hline HL Tau & $04: 31: 38.4253(0.0002)$ & $18: 13: 57.240(0.004)$ & 9 & $4.13(0.03)$ & $74.3(0.4)$ & $\sim 1750$ \\
\hline XZ Tau A & 04:31:40.09714 (0.00004) & $18: 13: 56.674(0.001)$ & 24 & $1.99(0.04)$ & $2.71(0.08)$ & $<54$ \\
\hline XZ Tau B & $04: 31: 40.08218(0.00007)$ & $18: 13: 56.845(0.001)$ & 24 & $1.30(0.04)$ & $1.83(0.08)$ & $<54$ \\
\hline
\end{tabular}

$1.3 \mathrm{~mm}$ (Band 6, 233.0 GHz) $35.1 \times 21.8 \mathrm{mas}$, P.A. $=+11^{\circ}$

HL Tau

04:31:38.42548 (0.00008)

$18: 13: 57.242(0.001)$

11

$6.48(0.03)$

$744.1(1.5)$

$\sim 1960$

$0.87 \mathrm{~mm}$ (Band 7, $343.5 \mathrm{GHz}) 29.9 \times 19.0 \mathrm{mas}$, P.A. $=-176^{\circ}$

HL Tau

04:31:38.4254 (0.0001)

$18: 13: 57.242(0.001)$

23

$11.56(0.07)$

$2140.8(3.7)$

$\sim 1960$

$1.0 \mathrm{~mm}\left(\right.$ Combined Band 6+7, 287.2 GHz) $33.5 \times 21.1 \mathrm{mas}$, P.A. $=+9^{\circ}$

HL Tau

04:31:38.42545 (0.00009)

$18: 13: 57.242(0.001)$

12

$9.79(0.04)$

$1441.5(1.8)$

$\sim 1960$

${ }^{a}$ For each band, the mean continuum frequency and synthesized beam is given after the wavelength. Uncertainties are given in parentheses after each quantity. All measurements are made from images corrected for primary beam attenuation.

${ }^{\mathrm{b}}$ The peak positions (and uncertainties) were measured using Gaussian fitting; for HL Tau the fit was restricted to the region inside the smallest radii gap.

${ }^{\mathrm{c}}$ For XZ Tau and LkH $\alpha 358$, the peak intensity, flux density, and size (or upper limit) were measured using Gaussian fitting. For HL Tau the peak intensity corresponds to the peak pixel value with an uncertainty of $3 \sigma$ (where $\sigma$ is given in the rms column). The flux density was measured within the $4 \sigma$ contour, and the uncertainty is [No. Independent Beams] ${ }^{0.5} \times 3 \sigma$. Uncertainties for these quantities do not include the $5 \%$ absolute flux uncertainty. The size of HL Tau was estimated from the length of the line passing through the continuum peak at a P.A. of $+138^{\circ}$ and where it crosses the $4 \sigma$ contour on each side of the disk.

\section{RESULTS}

Figure 1(a) demonstrates the complex outflow emission that fills the HL Tau region as traced by both atomic and molecular gas. The strong $\mathrm{H} \alpha$ and $[\mathrm{S}$ II] emission (observed with the Hubble Space Telescope) and weak blueshifted ${ }^{12} \mathrm{CO}(1-0)$ to the NE of HL Tau as well as the strong redshifted ${ }^{12} \mathrm{CO}(1-0)$ emission to the SW (observed with ALMA) are perpendicular to the disk, consistent with previous observations (see Section 1). Interestingly, the $\mathrm{H} \alpha,\left[\mathrm{S}_{\mathrm{II}}\right]$, and blueshifted ${ }^{12} \mathrm{CO}(1-0)$ emission to the SE of HL Tau parallel to the disk are difficult to reconcile with a simple disk/outflow scenario, suggesting that the blueshifted outflow has broken out of the parental core (Monin et al. 1996) or that there is another-as yet unidentified-driving source. Unfortunately, the ${ }^{12} \mathrm{CO}(1-0)$ data are missing significant flux (due to a lack of short spacings), and have insufficient sensitivity in the outer portions of the field of view to warrant deeper analysis of its properties. Figures 1(b) and (c) show zoomed in views of our serendipitous detections of XZ Tau (A and B) and LkH $\alpha 358$; no other continuum sources above the local $4 \sigma$ level were detected.

\subsection{HL Tau}

\subsubsection{Position and Proper Motion}

The fitted position for HL Tau in each of the ALMA images is given in Table 1. The phase calibrator positions are accurate to $<1$ mas and the positions are consistent between the three observed bands to better than 2 mas (consistent with dedicated LBC astrometry experiments; see ALMA I); thus, we assume 2 mas as the absolute ALMA position uncertainty. The position reported by Kwon et al. (2011) from $1.3 \mathrm{~mm}$ CARMA observations is $04^{\mathrm{h}} 31^{\mathrm{m}} 38^{\mathrm{s}} 418+18^{\circ} 13^{\prime} 57^{\prime \prime} 37$ (J2000, epoch 2009.08). The phase calibrator for CARMA observations $(\mathrm{J} 0510+1800)$ had a position accurate to better than 1 mas, and we assume an overall astrometric uncertainty of 5 mas for this measurement. Adding the two uncertainties in quadrature, the measured angular separation between the CARMA position and the $1.0 \mathrm{~mm}$ ALMA position (epoch 2014.83; Table 1) is $\Delta$ R.A. $=+106.1 \pm 5.6$ mas, $\Delta$ decl. $=-128.0 \pm 5.6$ mas. With a time span of 5.75 years, this separation amounts to a millimeter-derived proper motion of $v_{\text {R.A. }}=+18.5 \pm 1$ mas $\mathrm{yr}^{-1}$, and $v_{\text {Decl. }}=-22.3 \pm 1$ mas yr$^{-1}$. This result is in good agreement with the proper motion adopted by Guilloteau et al. (2011) based on 12 years of Plateau de Bure

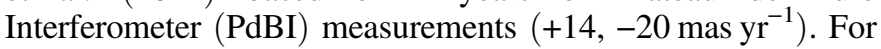
comparison, the weighted average of all optical and infrared proper motions for HL Tau listed in Vizier ${ }^{22}$ since 2000 (e.g., Roeser et al. 2010; Zacharias et al. 2013; Dias et al. 2014) is $v_{\text {R.A. }}=+2.0 \pm 2.4$ mas yr $^{-1}, v_{\text {Decl. }}=-20.9 \pm 2.4{\text { mas } \mathrm{yr}^{-1}}^{-1}$. Apparently, the optical/IR proper motion for HL Tau is reasonably accurate in decl., but is significantly underestimated in R.A. This discrepancy is not surprising given that the optical and even NIR emission from HL Tau is dominated by reflection nebulosity rather than stellar light (see, for example, Figure 1(a)).

\subsubsection{Continuum Emission}

Figures 2(a)-(c) show the HL Tau continuum at all three observed bands. These images reveal for the first time the remarkable morphology of the HL Tau protoplanetary disk, with a complex pattern of alternating bright and dark rings.

\footnotetext{
22 http://vizier.u-strasbg.fr/viz-bin/VizieR
} 

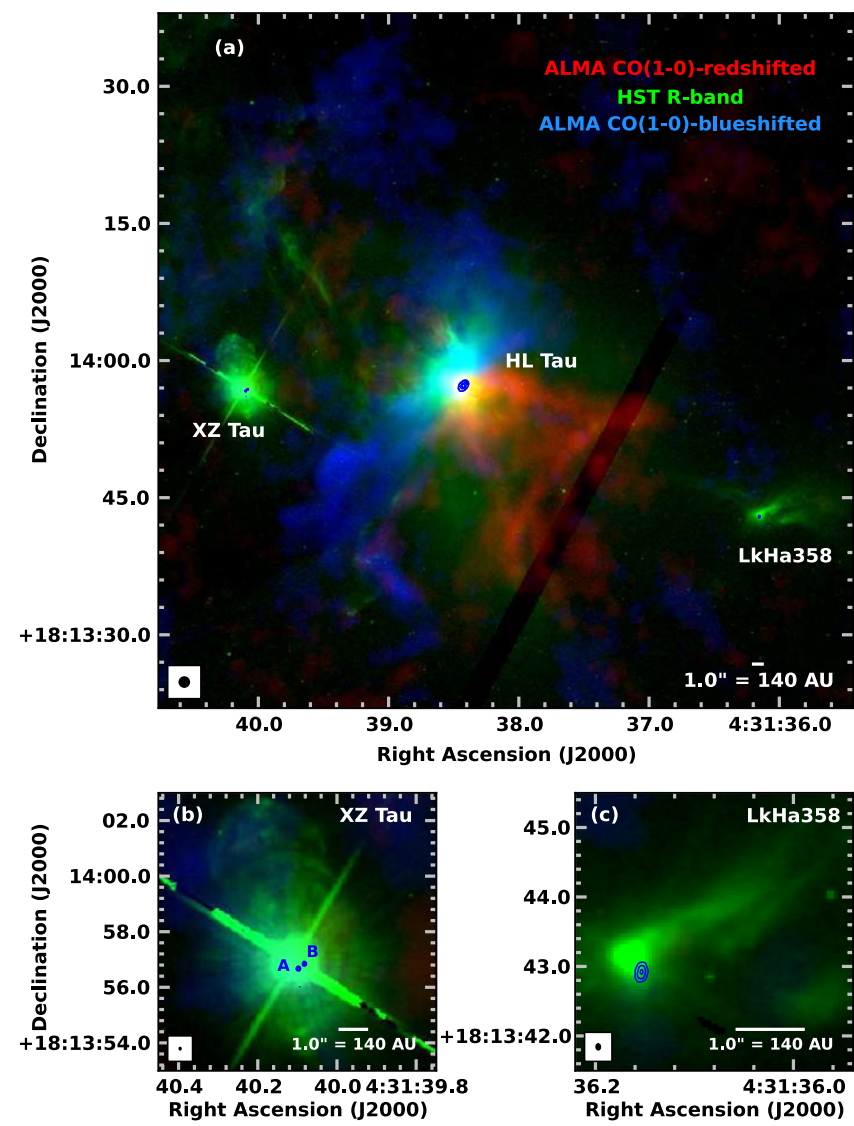

Figure 1. Panel (a) shows an overview of the HL Tau region with red and blue colors mapped to the integrated intensity of the redshifted $\left(7.25-20 \mathrm{~km} \mathrm{~s}^{-1}\right.$ ) and blueshifted $\left(0.0-6.0 \mathrm{~km} \mathrm{~s}^{-1}\right){ }^{12} \mathrm{CO}(1-0)$, respectively. The ALMA 1." 1 resolution ${ }^{12} \mathrm{CO}(1-0)$ images (synthesized beam shown in the lower left) have not been corrected for primary beam attenuation; the displayed field of view corresponds to the $\sim 15 \%$ power point. Green shows an HST R-band image (this band includes stellar continuum, $\mathrm{H} \alpha$, and $[\mathrm{S} \mathrm{II}]$ ). The darker diagonal stripe across the image (visible in some viewers) corresponds to a gap in the HST ACS WFC detector. Panels (b) and (c) show zoomed in views of XZ Tau (A and B) and LkH $\alpha 358$, respectively. All three panels show ALMA $2.9 \mathrm{~mm}$ primary beam corrected continuum contours overlaid in blue at $33 \times(4$, 14,19) $\mu \mathrm{Jy}$ beam $^{-1}$ (the corresponding synthesized beam is shown in the lower left of panels (b) and (c); also see Table 1). The HST image has been precessed to epoch 2014.83 using the proper motion derived in Section 3.1.1.

With the caveats of lower spatial resolution at $2.9 \mathrm{~mm}$ and reduced phase stability and hence image fidelity at $0.87 \mathrm{~mm}$, the agreement between the different wavelengths is excellent. Although the antenna configuration was not ideal (baselines were concentrated at lengths $<200 \mathrm{~m}$ and $>500 \mathrm{~m})$, a reasonably Gaussian synthesized beam was achieved as demonstrated in Figure 2(d) which shows the $1.3 \mathrm{~mm}$ pointspread function. The combined Band 6+7 image at $1.0 \mathrm{~mm}$ and its corresponding spectral index map are shown in Figures 2(e) and (f) (see the Appendix for details). Notably, while the ring structure remains clearly visible and much improved over the $0.87 \mathrm{~mm}$ data alone, the combined $1.0 \mathrm{~mm}$ image does not show some of the (potentially interesting) azimuthal changes in ring brightness seen in the individual images. These might be due to variations in dust opacity, but they could also just be the result of differences in the UV-coverage at the two wavelengths. Thus, we strongly caution against over-interpretation of subtle features unless verified by detailed modeling or future, even higher fidelity observations.
The continuum properties of the HL Tau disk are given in Table 1. A wide range of flux densities is available in the literature. Considering recent interferometric observations, $2.7 \mathrm{~mm}$ flux densities range from $94.1 \pm 0.9$ to $120 \pm 4 \mathrm{mJy}$ and $1.3 \mathrm{~mm}$ flux densities range from $700 \pm 10$ to $818 \pm 10.8$ mJy (Guilloteau et al. 2011; Kwon et al. 2011). Using the more precise $2.7 \mathrm{~mm}$ measurement and scaling by a spectral index $\alpha=3$, there is good agreement with the $2.9 \mathrm{~mm}$ ALMA measurement $(74.3 \pm 0.4 \mathrm{mJy})$. At $1.3 \mathrm{~mm}$, past observations bracket the total flux measured by ALMA (744.1 $\pm 1.5 \mathrm{mJy})$. At $0.87 \mathrm{~mm}$, only one interferometric measurement is available: $1300 \pm 300 \mathrm{mJy}$ (SMA with 2 " $1 \times 1$ × 10 resolution; Lumbreras \& Zapata 2014), but this is $60 \%$ smaller than the ALMA measurement of $2140.8 \pm 3.7 \mathrm{mJy}$. In contrast, previous bolometric James Clerk Maxwell Telescope measurements at $0.86 \mathrm{~mm}$ wavelength have obtained $2360 \pm 90 \mathrm{mJy}$ (Andrews \& Williams 2005), which is in much closer agreement with the ALMA data, especially considering the difference in angular resolution. This latter comparison suggests that only $\sim 10 \%$ of the emission is resolved out by the ALMA data.

Using the ALMA integrated flux densities from Table 1, the average spectral index is $\alpha=2.77 \pm 0.13$, with no significant curvature evident from 2.9 to $0.87 \mathrm{~mm}$, despite the expectation that the emission would be more optically thin at the longer wavelength. This result is likely a reflection of the fact that the central $\sim 10 \mathrm{AU}$ is both significantly brighter than the surrounding disk and is possibly optically thick even at $2.9 \mathrm{~mm}$. Additionally, there may be weak free-free contamination in the central region at $2.9 \mathrm{~mm}$ (see, for example, Wilner et al. 1996; Carrasco-González et al. 2009). For comparison, Andrews \& Williams (2005) find $\alpha=2.53 \pm 0.13$ based on a compilation of primarily bolometer measurements between 1.3 and $0.35 \mathrm{~mm}$, suggesting the average spectral index does eventually flatten at shorter wavelengths.

The HL Tau disk is viewed on the sky inclined with respect to our line of sight (defined as $i=0^{\circ}$ for face-on) and rotated by an amount defined by the P.A. measured from north to east. In order to constrain these parameters we first followed the standard practice of fitting a Gaussian to the visibility data. Using baselines $<1000 \mathrm{~m}\left(\sim 0 .{ }^{\prime \prime} 2\right)$, we obtain $i=46.2 \pm 0.2$ and P.A. $=138.2 \pm 0.2$ in good agreement with past $U V$ plane estimates (Guilloteau et al. 2011; Kwon et al. 2011). However, the fantastic fidelity and angular resolution afforded by the ALMA images allow us to go further and explore the properties of the rings independently in the image plane.

\subsubsection{Spatially Resolved Disk Geometry and Spectral Index}

Upon visual inspection, one can plausibly identify seven pairs of "bright" and "dark" rings in the $1.0 \mathrm{~mm}$ image; we label these rings B1..B7 and D1...D7, respectively. As a first step, we assumed that the rings trace circular orbits around a common center position defined by the $1.0 \mathrm{~mm}$ continuum peak position (see Table 1), having the $i$ and P.A. derived in Section 3.1.2. Then, using a cross-cut along the major axis of the image, we determine approximate semimajor axes for the 14 rings.

After overlaying these zeroth-order ellipses on the image, it was apparent that while approximately correct, these are not a good fit to the rings in detail. To refine the parameters, we defined a discrete set of points along each zeroth-order ellipse, at a Nyquist sampled interval with respect to the synthesized 


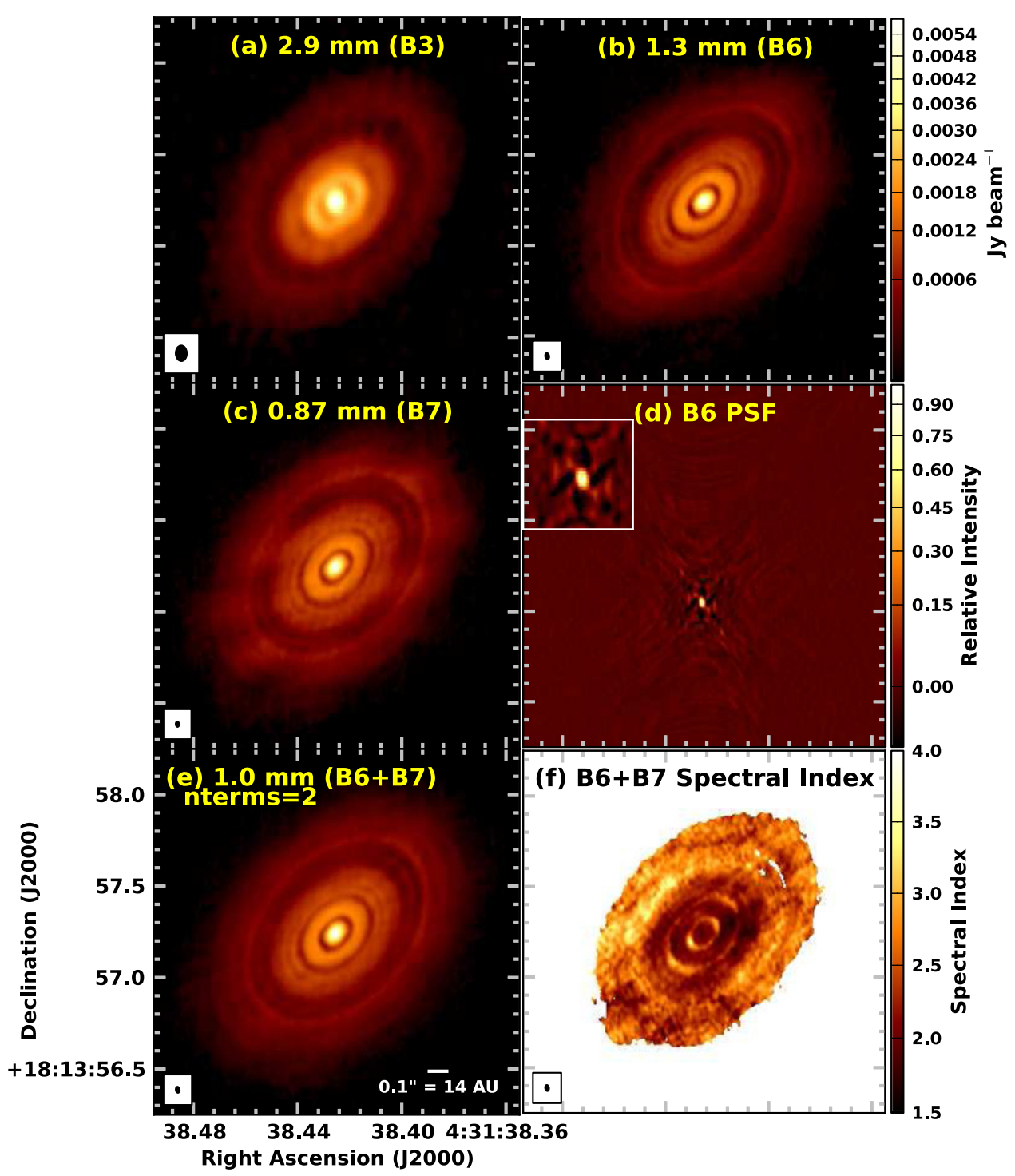

Figure 2. Panels (a), (b), and (c) show 2.9, 1.3, and $0.87 \mathrm{~mm}$ ALMA continuum images of HL Tau. Panel (d) shows the $1.3 \mathrm{~mm}$ PSF for the same FOV as the other panels as well as an inset with an enlarged view of the inner 300 mas centered on the PSF's peak (the other bands show similar patterns). Panels (e) and (f) show the image and spectral index maps resulting from the combination of the 1.3 and $0.87 \mathrm{~mm}$ data. The spectral index $(\alpha)$ map has been masked where $\alpha / \alpha_{\text {error }}<4$. The synthesized beams are shown in the lower left of each panel; also see Table 1 . The range of the color bar shown for panel (b) at $1.3 \mathrm{~mm}$ corresponds to $-2 \times$ rms to $0.9 \times$ the image peak using the values in Table 1 . The color scales for panels (a), (c), and (e) are the same except using the values of rms and image peak corresponding to each respective wavelength in Table 1.

beam. Then the position of each point was moved to the nearest local radial maximum (or minimum for dark rings). To avoid regions where the rings become less distinct, points were discarded if they moved outside the nominal width of the individual rings $(5$ to $8 \mathrm{AU})$. Eight rings retained $>55 \%$ of the points, to which we subsequently fit an ellipse, including its center position, using a Markov Chain Monte Carlo (ForemanMackey et al. 2013). The results are listed in Table 2, with the full range of parameters given for the eight most distinct rings, and just the semimajor axis for the others. It seems likely that the "gap," "enhancement," and "clump" observed in VLA 1.3 and $0.7 \mathrm{~cm}$ images (Greaves et al. 2008; Carrasco-González et al. 2009) at 10, 20, and $55 \mathrm{AU}$ along the major axis of the disk correspond to the $\mathrm{D} 1, \mathrm{~B} 1$, and the combined emission from the $\mathrm{B} 2$ to $\mathrm{B} 4$ rings, respectively.

The weighted average of the best-fit inclination and P.A. for the eight fitted rings yields $i=46.72 \pm 0.05$ and P.A. $=138^{\circ} .02 \pm 0.07$, consistent with the constraints found for the average disk geometry over large scales. However, the bestfit ellipses have their centers offset with respect to the peak of the $1.0 \mathrm{~mm}$ emission, as can be seen in the equatorial offsets reported in Table 2 . These offsets are statistically significant for all but the innermost ring (D1). Interestingly, the magnitude of the position offset increases with orbital distance from the center.

Using the weighted average inclination and P.A., we have deprojected the combined $1.0 \mathrm{~mm}$ visibility data into a circularly symmetric, face-on equivalent view (see Figure 3 (a)). We have also extracted cross-cuts at an angle of $138^{\circ}$ from both the $1.0 \mathrm{~mm}$ continuum image and the spectral index map shown in Figures 2(e) and (f). These cross-cuts are shown in Figures $3(\mathrm{~b})$ and $(\mathrm{c})$. The variation in intensity between the bright and dark rings is readily apparent. Considering only the fully characterized rings, the largest average intensity contrast is between the first pair with D1 being $46 \%$ less bright than B1, and the smallest contrast is between the 5th pair with D5 being 
Table 2

Properties of Bright and Dark Rings ${ }^{\mathrm{a}}$

\begin{tabular}{|c|c|c|c|c|c|}
\hline \multirow{2}{*}{$\begin{array}{l}\text { Ring } \\
\text { Name }\end{array}$} & \multicolumn{2}{|c|}{ Position Offset $^{\mathrm{b}}$} & \multirow{2}{*}{$\begin{array}{c}\text { Semi-major } \\
(\mathrm{AU})\end{array}$} & \multirow{2}{*}{$\begin{array}{c}\text { Inclination } \\
\left({ }^{\circ}\right)\end{array}$} & \multirow{2}{*}{$\begin{array}{l}\text { P.A. } \\
\left({ }^{\circ}\right)\end{array}$} \\
\hline & R.A.(mas) & Decl.(mas) & & & \\
\hline D1 & $-2.0 \pm 0.9$ & $-1.9 \pm 0.9$ & $13.2 \pm 0.2$ & $44.9 \pm 1.1$ & $137.2 \pm 1.8$ \\
\hline B1 & $-0.7 \pm 0.7$ & $-4.9 \pm 0.7$ & $20.4 \pm 0.1$ & $46.0 \pm 0.5$ & $138.2 \pm 0.9$ \\
\hline D2 & $-0.9 \pm 0.5$ & $-3.0 \pm 0.6$ & $32.3 \pm 0.1$ & $45.5 \pm 0.3$ & $138.1 \pm 0.5$ \\
\hline B2 & $-1.6 \pm 0.5$ & $-7.9 \pm 0.6$ & $38.1 \pm 0.1$ & $46.5 \pm 0.2$ & $137.3 \pm 0.4$ \\
\hline D3 & $\ldots$ & $\ldots$ & $\sim 42$ & $\ldots$ & $\ldots$ \\
\hline B3 & $\ldots$ & $\ldots$ & $\sim 47$ & $\ldots$ & $\ldots$ \\
\hline D4 & $\ldots$ & $\ldots$ & $\sim 50$ & $\ldots$ & $\ldots$ \\
\hline B4 & $\ldots$ & $\ldots$ & $\sim 55$ & $\ldots$ & $\ldots$ \\
\hline D5 & $-1.8 \pm 0.4$ & $3.4 \pm 0.5$ & $64.2 \pm 0.1$ & $45.8 \pm 0.1$ & $138.9 \pm 0.2$ \\
\hline B5 & $-0.8 \pm 0.5$ & $7.5 \pm 0.6$ & $68.8 \pm 0.1$ & $46.6 \pm 0.1$ & $138.8 \pm 0.2$ \\
\hline D6 & $-4.7 \pm 0.5$ & $8.3 \pm 0.5$ & $73.7 \pm 0.1$ & $47.9 \pm 0.1$ & $137.0 \pm 0.2$ \\
\hline B6 & $-8.6 \pm 0.4$ & $12.3 \pm 0.4$ & $81.3 \pm 0.1$ & $46.8 \pm 0.1$ & $137.9 \pm 0.1$ \\
\hline D7 & $\ldots$ & $\ldots$ & $\sim 91$ & $\ldots$ & $\ldots$ \\
\hline B7 & $\ldots$ & $\ldots$ & $\sim 97$ & $\ldots$ & $\ldots$ \\
\hline
\end{tabular}

\footnotetext{
${ }^{a}$ Rings with a complete set of parameters were calculated assuming circular orbits that are tilted by the inclination and rotated in the sky by the P.A. using the method described in Section 3.1.3. Rings with only a semimajor axis entry were estimated directly from the image and have an uncertainty of the order of 1 AU.

${ }^{\mathrm{b}}$ Offset positions are with respect to the fitted $1.0 \mathrm{~mm}$ peak position in Table 1 .
}

$15 \%$ less bright than B5. Such a drop in intensity could be due to a reduction in dust temperature, column density, or grain emissivity, or a combination thereof. This figure also demonstrates the general trend of having very high signal-tonoise ratio $(\mathrm{S} / \mathrm{N})$ on the estimate of $\alpha$ in the (brighter) inner parts of the disk with increasing uncertainty as the disk intensity decreases. Interestingly, the minimum $\alpha$ does not occur precisely at the continuum peak position, but is instead offset by $\sim 1.5 \mathrm{AU}(\sim 10.7 \mathrm{mas})$ along the major axis to the SE. The origin of this offset is unknown.

As shown in Figures 3(b) and (c), each dark ring corresponds to a local maximum in the spectral index, while each bright ring corresponds to a local minimum. We do not find a gradual decrement of the spectral index with radius reported in other lower mass protoplanetary systems, albeit with reduced angular resolution (e.g., Guilloteau et al. 2011; Pérez et al. 2012). The central peak, the B1 ring, and the B6 ring, show a spectral index $\alpha \sim 2$ indicative of optically thick dust emission (within $3 \sigma$ ). The observed $T_{B}$ (see Figure $3(\mathrm{~d})$ ) provides a strict lower limit on the physical temperature at a given angular resolution. In the limit where the emission is optically thick, $T_{B}$ provides a measure of the physical temperature of the material where $\tau \approx 1$. The $T_{B}$ for the optically thick continuum peak and azimuthally averaged values for the optically thick B1 and B6 rings are 212.4 \pm $0.8 \mathrm{~K}, 59 \pm 3 \mathrm{~K}$, and $24 \pm 2 \mathrm{~K}$ (corresponding intensities are $9.79 \pm 0.04,2.48 \pm 0.12$, and $\left.0.85 \pm 0.06 \mathrm{mJy} \mathrm{beam}^{-1}\right)$, at radii of $\sim 0,20$, and $81 \mathrm{AU}$, respectively. As shown in Figure $3(\mathrm{~d})$, the observed radial decrease in $T_{B}$ for all the bright rings can be roughly characterized by a power law with an exponent of $\sim-0.65$. For comparison, Guilloteau et al. (2011) found $T_{B}=25 \mathrm{~K}$ at $55 \mathrm{AU}$ from $\sim 1^{\prime \prime}$ resolution PdBI data in good agreement with that predicted from the ALMA $T_{B}$ analysis. However, the observed $T_{B}$ power law should not be mistaken for a model of the physical dust temperature because much of the disk does not have a spectral index consistent with optically thick emission.

The standard model used for circumstellar disks consists of a surface layer of grains directly heated by stellar photons which subsequently illuminates and heats the dust in the lower layers and the midplane (Chiang \& Goldreich 1997). While the surface layer is optically thick to stellar photons, it is optically thin at millimeter wavelengths. Thus, the brightness temperature observed by ALMA in the optically thick regions should correspond to the physical temperature at the $\tau \approx 1$ surface, which will be somewhere between the surface temperature $\left(T_{S}\right)$ and the midplane temperature $\left(T_{m}\right)$. The observed $T_{B}$ in the central peak and the B1 ring do indeed lie between the values of $T_{s}$ and $T_{m}$ at those radii predicted from the Kwon et al. (2011) "thick-disk" model that best fits the full HL Tau SED. Furthermore, the $T_{B}$ of all of the dark rings falls below $T_{m}$, consistent with the dark rings being optically thin. The $T_{B}$ in the B6 ring is somewhat below the $T_{m}$ prediction of $33 \mathrm{~K}$ at 81 AU (despite having $\alpha \sim 2$ ). One possible interpretation for this behavior is that B6 is not optically thick but instead has very low $\beta$. However, we note that the Kwon et al. (2011) "thin-disk" model, which provides the best match to the previous millimeter data alone (due to dust settling to the midplane), predicts $T_{m}$ values that fall below the observed $T_{B}$ (already a lower limit on the physical temperature) in all of the dark rings. Clearly, more sophisticated modeling of HL Tau's physical dust temperature distribution that includes the radial intensity and spectral index variations as well as radial changes in the scale-height will be fruitful.

As shown in Figures 3(b) and (d), the dark rings are not completely devoid of emission, and the spectral index at their location differs from the optically thick expectation of $\alpha=2$, with larger $\alpha$ values ranging from $\sim 2.3$ to 3.0. This observation rules out the possibility that the reduction in emission is due solely to radial temperature variations. Instead, these regions are likely optically thin, consistent with their lower observed $T_{B}$. An estimate for the value of the dust opacity spectral index $\beta$ can be derived in the optically thin limit for warm dust where $\beta=\alpha-2$ (Beckwith \& Sargent 1991). We estimate $\beta \sim 0.8$ for D1, $\beta \sim 0.7$ for D2, $\beta \sim 0.6$ for D5, and $\beta \sim 0.3$ for D6, consistent with some amount of grain growth and evolution inside these dark rings. However, further physical modeling of the disk dust continuum emission is needed to confirm these estimates. Finally, we caution that observations that cannot resolve the HL Tau morphology will combine emission from 


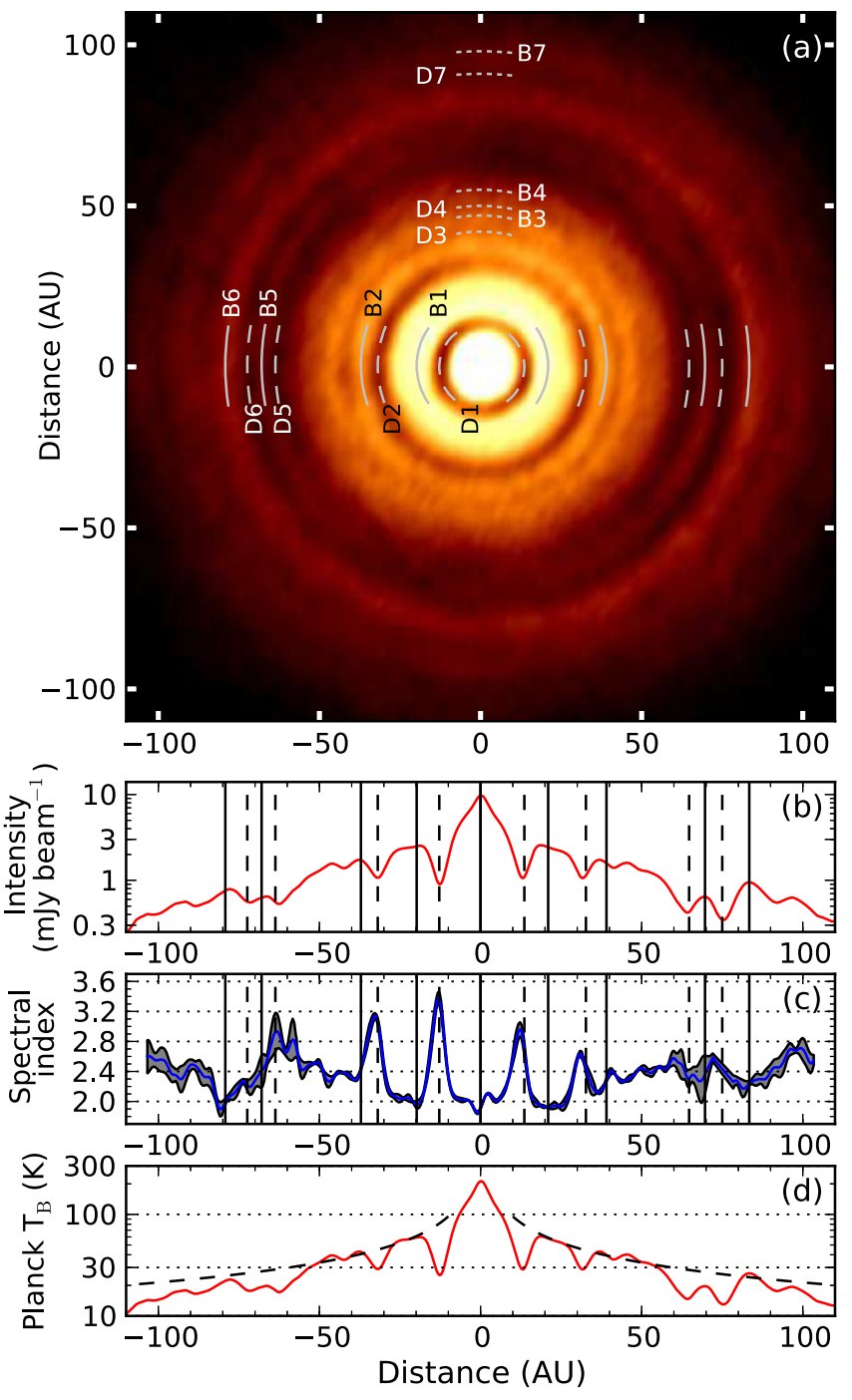

Figure 3. Panel (a) shows the deprojected $1.0 \mathrm{~mm} \mathrm{B6+B7}$ image of HL Tau (see Section 3.1.3); the angular resolution is $38.6 \times 19.3$ mas (P.A . $-20.7)$. Rings for which a full range of ellipse parameters could be fit are labeled horizontally (solid and dashed lines), while the less distinct rings are labeled vertically (dotted lines). Panels (b) and (c) show cross-cuts at P.A. $=138^{\circ}$ through the continuum peak of the $1.0 \mathrm{~mm}$ continuum and spectral index images shown in Figures 2(e) and (f) (positive values of distance correspond to the SE portion of Figure 2(e)). In panel (c) the gray region delineates the statistical $1 \sigma$ spectral index uncertainty; it does not account for the absolute flux uncertainty. For the fully fitted rings, panels (b) and (c) show dashed lines for the locations of dark rings and solid lines for bright rings. The fitted offsets from the continuum peak (Table 2) have been taken into account. Panel (d) shows the same cross-cut as panel (b) but on a Planck brightness temperature $\left(T_{B}\right)$ scale. Panels (b) and (d) are shown on a log scale on the $y$ axis. The dashed curve on panel (d) shows a representative power law for $T_{B}$ as a function of radius with an exponent of -0.65 extending from the B1 peak; it is not a fit.

optically thick and thin regions, driving any derived values of dust opacity spectral index to be lower than reality.

\subsubsection{Compact Spectral Lines}

At 1". 1 resolution, the $\mathrm{HCO}^{+}(1-0)$ emission shows a morphology similar to the ${ }^{12} \mathrm{CO}(1-0)$ shown in Figure 1(a), albeit somewhat less extended. Interestingly, it also shows a barely resolved velocity gradient across the HL Tau disk itself, though it is confused with the surrounding outflow gas, especially to the NE of the disk. Fortunately, at 0 "' 25 resolution most of the outflow emission is resolved out, making it possible to spatially resolve the morphology of the HL Tau molecular gas disk for the first time. The 0. !' 25 resolution $\mathrm{HCO}^{+}(1-0)$ channel maps shown in Figure 4 reveal: (1) a notable deficit of blueshifted versus redshifted emission, (2) $\mathrm{HCO}^{+}(1-0)$ absorption near the systemic velocity $6.5-7.0 \mathrm{~km} \mathrm{~s}^{-1}$, (3) a roughly Keplerian velocity distribution with a detectable radial velocity range of 2.0 to $12.0 \mathrm{~km} \mathrm{~s}^{-1}$, and (4) comparable gas and continuum disk sizes (at least at the current sensitivity level). To further explore points (1) and (2), Figure 5 shows spectra from the 1 "! 1 resolution cubes toward the continuum peak. All of the lines show absorption at $6.0-7.0 \mathrm{~km} \mathrm{~s}^{-1}$, indeed $\mathrm{CN}$ and $\mathrm{HCN}(1-0)$ are only detected in absorption. Additionally, the absorption is non-Gaussian in shape, instead showing a gradual increase in absorption on the blueshifted side compared to a steeper rise on the redshifted side. As shown in Figure 1(a), the blueshifted outflow emerges from the NE of HL Tau and, based on the continuum disk orientation, propagates toward us at $i \approx 47^{\circ}$. Self-absorption by this outflowing gas is likely responsible for both the non-Gaussian line shape and the deficit of observable blueshifted disk emission. The deepest absorption for the $\mathrm{CN}$ and HCN transitions occurs at an LSRK velocity of $7.0 \pm 0.2 \mathrm{~km} \mathrm{~s}^{-1}$, which we take to be the rest velocity of the system.

Under the assumption of circular Keplerian motion and noting that the velocity extrema of $\mathrm{HCO}^{+}(1-0)$ emission occurs at $\Delta V \pm 5 \mathrm{~km} \mathrm{~s}^{-1}$ (see Figure 4) from the systemic velocity $\left(7.0 \mathrm{~km} \mathrm{~s}^{-1}\right)$ at a radius of $\sim 25 \mathrm{AU}$, we find that with $i=47^{\circ}$, the enclosed mass is $\sim 1.3 M_{\odot}$. This value is near the high end of the range previously reported for HL Tau (see Section 1). However, it is clear that even on the less absorbed redshifted side, the velocity pattern is not so simple and may, for example, have a contribution from infall (see, for example, Gómez \& D'Alessio 2000). Future detailed radiative transfer analysis coupled with a physical model will be required to reproduce the complex $\mathrm{HCO}^{+}(1-0)$ absorption and emission toward the HL Tau disk in order to obtain a more accurate kinematic stellar mass.

\subsection{XZ Tau $2.9 \mathrm{~mm}$ Continuum}

At $2.9 \mathrm{~mm}$ we resolve the known multiple system XZ Tau into two components, A and B (Figure 1(b) and Table 1; also see Forgan et al. 2014 and Carrasco-González et al. 2009), separated by $273 \pm 1$ mas at a P.A. of $128.7 \pm 0.5$. This separation is $8 \%$ smaller than predicted by Forgan et al. (2014) for a circular, face-on orbit (296 \pm 1 mas), particularly in R.A. This is likely an indication that the orbit is not face-on, but will require future observations to confirm and quantify. Like Forgan et al. (2014), we find no evidence for component " $C$ " (a putative third star) reported by Carrasco-González et al. (2009) at $7 \mathrm{~mm}$. Using the $2.9 \mathrm{~mm}$ flux densities from Table 1 and the JVLA $7 \mathrm{~mm}$ flux densities from Forgan et al. (2014), we find spectral indices of $+1.8 \pm 0.5$ for both XZ Tau A and $\mathrm{B}$, suggesting both have a free-free component in addition to dust emission (see also Carrasco-González et al. 2009).

\subsection{LkHo358 $2.9 \mathrm{~mm}$ Continuum}

At $2.9 \mathrm{~mm}$, we have resolved the $\mathrm{LkH} \alpha 358$ disk for the first time in the millimeter continuum to a size of only $21 \pm 1 \mathrm{AU}$ with an inclination angle of $56 \pm 2^{\circ}$ at a P.A. $=170 \pm 3^{\circ}$ (see Figure 1(c) and Table 1). Schaefer et al. (2009) used PdBI to 


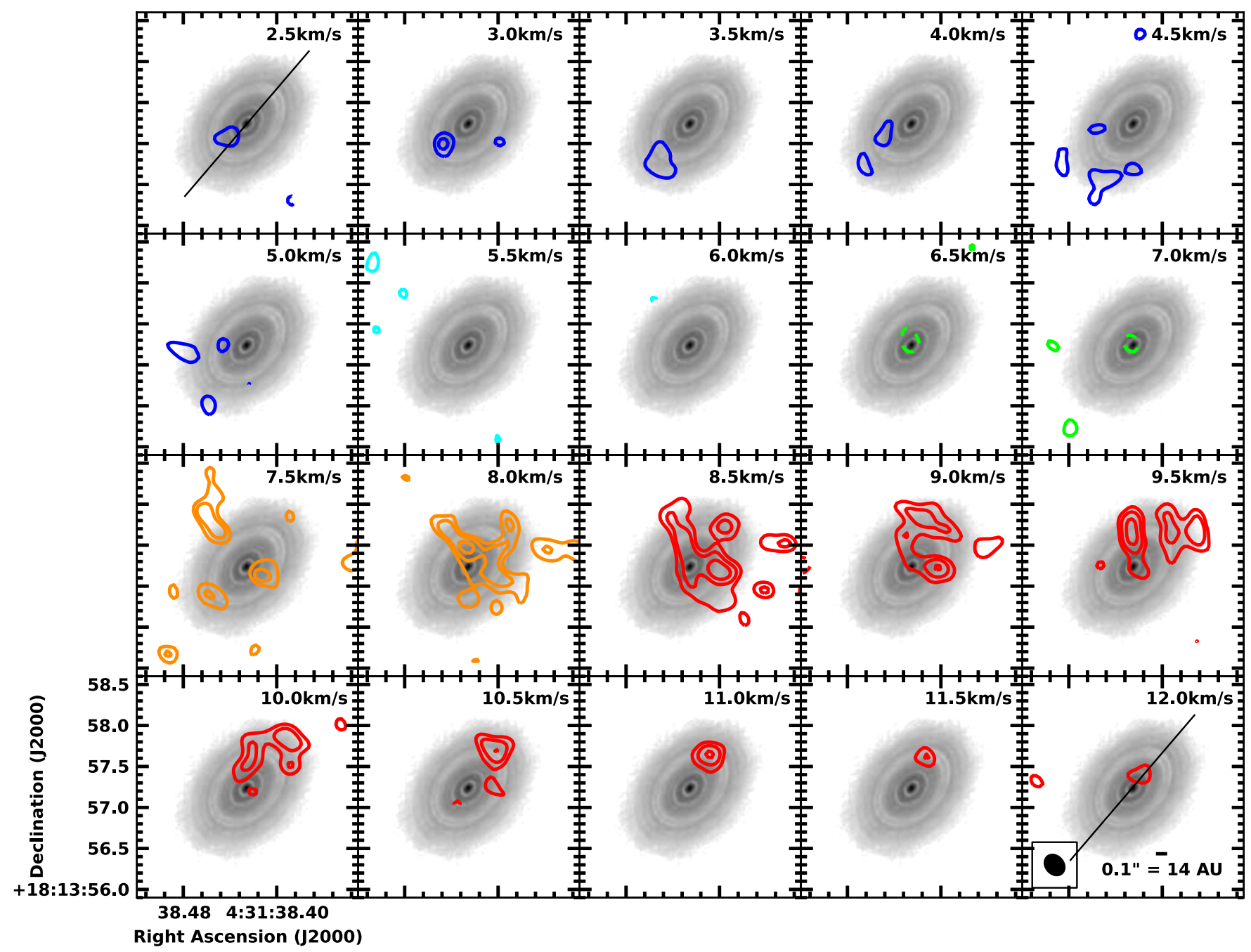

Figure 4. Contours show channel maps of the $\mathrm{HCO}^{+}(1-0)$ emission with $\sim 0.25$ angular resolution and $0.5 \mathrm{~km} \mathrm{~s}^{-1}$ velocity resolution overlaid on the $1.0 \mathrm{~mm} \mathrm{~B} 6+\mathrm{B} 7$ continuum image in grayscale. The LSRK velocity of the emission is shown in the upper right corner of each panel. The contour levels are at $1.9(1 \sigma) \times(-3.5,3.5,5.0$, 7.0) $\mathrm{mJy}_{\text {beam }}^{-1}$ and the color indicates the change from blue- to redshifted emission. Negative contours are shown as dashed. The cross-cut position angle of $+138^{\circ}$ is shown on the 2.5 and $12.0 \mathrm{~km} \mathrm{~s}^{-1}$ panels and the $\mathrm{HCO}^{+}(1-0)$ synthesized beam is shown in the lower left corner of the last panel.

observe ${ }^{12} \mathrm{CO}(2-1)$ with a resolution of $1 . " 38 \times 0 . " 83$ toward this source, obtaining an inclination of $28 \pm 9^{\circ}, V_{100} \sin i=1.35$ $\pm 0.04 \mathrm{~km} \mathrm{~s}^{-1}$ (the radial velocity at a radius of $100 \mathrm{AU}$ ), and a dynamical mass of $0.5-2 M_{\odot}$. These authors note that the inclination angle is not well constrained by the PdBI data. If we assume instead that the molecular gas has the same inclination as the continuum, the dynamical mass is significantly smaller: $0.3 \pm 0.1 M_{\odot}$. The ALMA $2.9 \mathrm{~mm}$ integrated flux density measured for $\mathrm{LkH} \alpha 358(3.7 \pm 0.1 \mathrm{mJy})$ is in reasonable agreement with that measured by Schaefer et al. (2009) at $2.7 \mathrm{~mm}: 4.0 \pm 0.6 \mathrm{mJy}$.

\section{DISCUSSION}

What is the nature of the remarkable pattern of bright and dark rings in the continuum emission from the HL Tau protoplanetary disk? Here we highlight four key observational findings. First, the correlated radial structure of higher spectral index and lower brightness temperature in the dark rings, relative to the bright rings, demonstrates that the optical depth must be lower in the dark rings. Second, the fact that the centers of nearly all of the rings are significantly offset from the dust continuum peak provides compelling evidence that the rings are not circular as assumed, but rather have some eccentricity with HL Tau at one focus. Third, the magnitude of the offset increases with ring radius, which is tantalizingly congruent with the observed increase in orbital eccentricity for exoplanets at large orbital radii (Butler et al. 2006; Shen \& Turner 2008). Fourth, several of these rings appear to be in resonances with each other. Assuming Keplerian orbits and neglecting the mass between the rings, the first four dark rings have orbital periods in the proportion D1:D2:D3:D4 = 1:4:6:8. There also appear to be resonances between bright and dark rings, with D2 in a 2:1 resonance with $\mathrm{B} 1$ and in a 1:4 resonance with $\mathrm{B} 6 .^{23}$ These mean motion resonances (MMRs) are calculated to be within the confidence intervals obtained on the semimajor axes. Given the precise constraints obtained on the orbital radii of the bright and dark rings, the $\mathrm{n}: \mathrm{m}$ resonances found here have uncertainties between 0.01 and 0.09 , making these resonance ratios unlikely to arise from random chance.

\footnotetext{
${ }^{23}$ Other bright rings are close to but not exactly at the following integer resonance ratios: D2:B2:B3 are near a 3:4:5 resonance, while D2:B5 are close to a $1: 3$ resonance.
} 


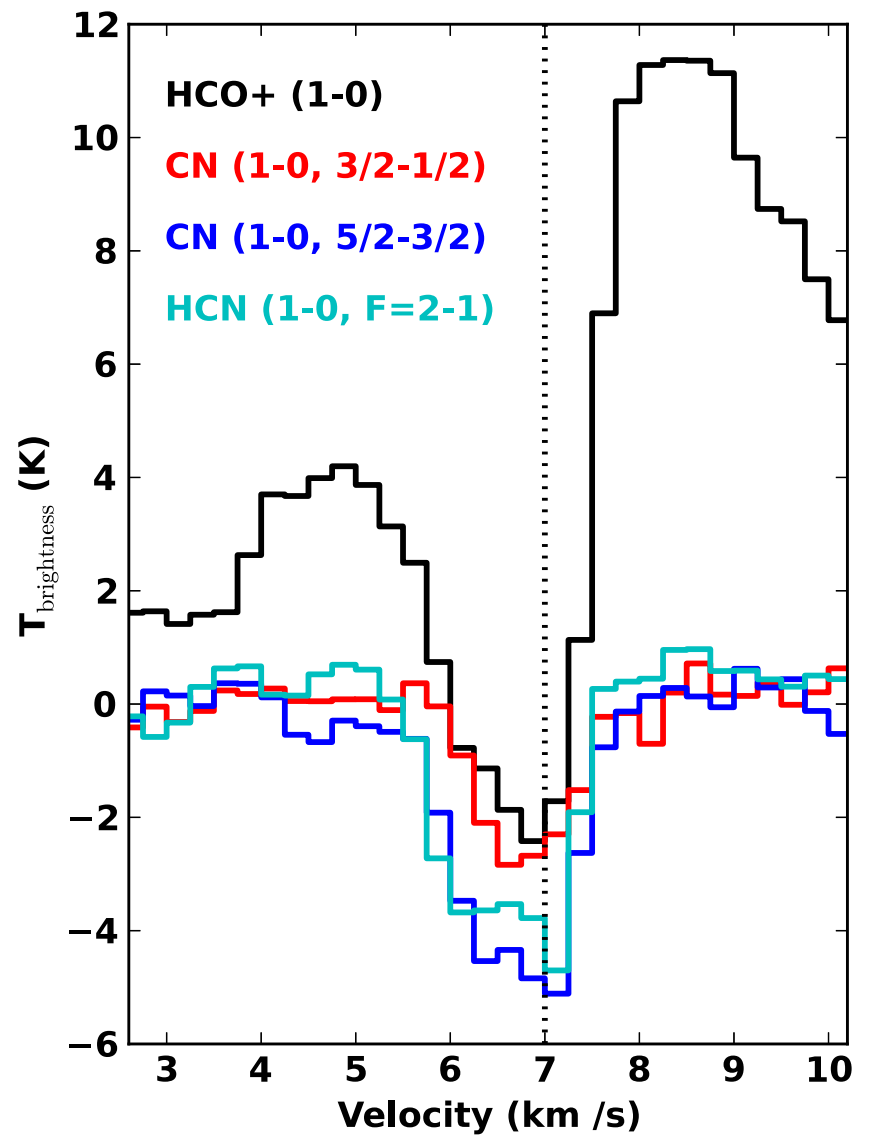

Figure 5. Continuum-subtracted spectra with an angular resolution of 1." 1 from the indicated transitions toward the HL Tau continuum peak. The adopted LSRK systemic velocity of $7 \mathrm{~km} \mathrm{~s}^{-1}$ is indicated by the vertical dotted line. For comparison, the $2.9 \mathrm{~mm}$ peak continuum surface brightness at 1. " 1 resolution is $5.75 \pm 0.01 \mathrm{~K}$.

Although multi-planetary systems show a diverse architecture ${ }^{24}$ and many pairs of exoplanets are far from being in MMR (Lissauer et al. 2011), a growing number of stable systems do exist in or near MMR (Zhang et al. 2014). Gravitational interactions between planets and their parent disks can lead to resonant and near-resonant systems (see Baruteau \& Papaloizou 2013 and references therein). Collectively, these four independent lines of evidence suggest that the observed dark rings are low column density gaps in the disk material arising from the process of planet formation.

\section{CONCLUSIONS}

In this paper, we present multi-wavelength ALMA images of the HL Tau protoplanetary disk at resolutions as fine as a few AU. We derive precise measurements of the disk inclination and P.A. as well as the proper motion of the central protostar. We also show the first molecular line observations to spatially resolve the disk kinematics. Under the assumption of Keplerian rotation, the mass of the central star is $M_{*} \sim 1.3 M_{\odot}$, but further refinement will require detailed radiative transfer calculations. The remarkable pattern of bright and dark circumstellar rings in the continuum images exhibits a

\footnotetext{
${ }^{24}$ Note that HR8799 is the only currently known multi-exoplanet system spanning a range of semimajor axes comparable to the gaps in the HL Tau disk (see Goździewski and Migaszewski 2014 and references therein). The planets in other multiple systems orbit within $\sim 5 \mathrm{AU}$ of the host star.
}

corresponding structure in the spectral index image, revealing that the dust in the central core and several of the bright rings are optically thick, while the dust in the dark rings - which are not completely empty-shows evidence of grain growth. Several characteristics of these rings, including an increase in eccentricity with radius and numerous resonances, suggest that the dark rings are gaps arising from the process of planet formation. Modeling of the disk temperature and density structure in both gas and dust, coupled with planet formation processes will be crucial to the goal of reproducing HL Tau's morphology from theory (e.g., Dong et al. 2014). These transformational ALMA observations of HL Tau herald a new era in the study of protoplanetary disks that promises to unearth the architecture of extrasolar multi-planetary systems during their epoch of formation and thereby illuminate the origin of our own solar system.

This paper makes use of the following ALMA data: ADS/ JAO.ALMA\#2011.0.00015.SV. ALMA is a partnership of ESO (representing its member states), NSF (USA), and NINS (Japan), together with NRC (Canada), NSC and ASIAA (Taiwan), and KASI (Republic of Korea), in cooperation with the Republic of Chile. The Joint ALMA Observatory is operated by ESO, AUI/NRAO and NAOJ. The National Radio Astronomy Observatory is a facility of the National Science Foundation operated under cooperative agreement by Associated Universities, Inc. This research made use of Astropy, a community-developed core Python package for Astronomy (Astropy Collaboration et al. 2013), as well as the VizieR catalogue access tool, CDS, Strasbourg, France. This paper also makes use of observations made with the NASA/ESA Hubble Space Telescope, obtained from the Hubble Legacy Archive, which is a collaboration between the Space Telescope Science Institute (STScI/NASA), the Space Telescope European Coordinating Facility (ST-ECF/ESA), and the Canadian Astronomy Data Centre (CADC/NRC/CSA).

Facility: ALMA.

\section{APPENDIX \\ IMAGING DETAILS}

The data were calibrated in CASA 4.2.2 using the same procedure adopted for standard science operations, with two exceptions. An imprecise position was inadvertently used for the phase calibrator J0431+2037 employed for the 2.9 and $1.3 \mathrm{~mm}$ data. The shift was small ( $\sim 15 \mathrm{mas})$, but noticeable when compared to the $0.87 \mathrm{~mm}$ data, for which a precise position for J0431+1731 was employed. The position of J0431 +2037 was corrected to $(\mathrm{J} 2000) 04^{\mathrm{h}} 31^{\mathrm{m}} 03.76137^{\mathrm{s}}+20^{\circ} 37^{\prime}$ $34.2649^{\prime \prime}$ before calibration, after which excellent position agreement for HL Tau was achieved across all three bands (see fitted positions in Table 1). Both final phase calibrator positions were taken from http://astrogeo.org/vlbi/solutions/rfc_2012b/ rfc_2012b_cat.txt. The small shifts in position due to proper motion over the month-long observing span have been ignored. Additionally, the final LBC antenna position corrections were applied before the data were calibrated (see ALMA I). For each execution, the bandpass and absolute flux calibrators were dynamically chosen by the ALMA online system and alternated between the frequently monitored quasars J04230120 and J0510+1800 depending on the LST start time (see footnote 22). Based on comparison of the resulting calibrated phase calibrator flux densities across the many executions for 
each band, we estimate that the absolute flux calibration is good to within $5 \%$ for the final combined data at each wavelength.

The continuum images for all three bands were made using multi-frequency synthesis, and the visibility weighting employed was mid-way between natural and uniform (i.e., CASA clean parameter robust $=0.0$ ). Individually, the $1.3 \mathrm{~mm}$ and $0.87 \mathrm{~mm}$ data have a fractional bandwidth small enough $(<10 \%)$ to ignore spectral index effects in the imaging. In contrast, the range of continuum spws available for the $2.9 \mathrm{~mm}$ image (90.8-102.9 GHz), if all were combined, would require accounting for at least a linear spectral index slope across the band given the expected range of dust spectral indices $\alpha=2-4$ $\left(S_{\nu} \propto \nu^{\alpha}\right)$. However, inclusion of the spectral slope requires good $\mathrm{S} / \mathrm{N}$ at both frequency extrema of the calculation (Rau \& Cornwell 2011), a condition the $2.9 \mathrm{~mm}$ data did not fulfill since four of the five available continuum spws were clustered at $\sim 102 \mathrm{GHz}$. Thus, the $2.9 \mathrm{~mm}$ continuum image was created excluding the $90.8 \mathrm{GHz}$ spw and without accounting for the spectral index. To make an image with the highest possible combination of fidelity and angular resolution we also made a combined Band $6+7(1.0 \mathrm{~mm})$ image (fractional bandwidth $44 \%$ ) accounting for spatial variations in the spectral index (CASA clean parameter nterms $=2$ ). In this case, the $\mathrm{S} / \mathrm{N}$ is high at both extrema and the resulting image and spectral index map are reliable (the derived $\alpha$ have a statistical $\mathrm{S} / \mathrm{N}$ of up to several hundred). Additionally, we used the multi-scale imaging option (Cornwell 2008) for all three bands with scales of 0,5 , and 15 , approximately corresponding to 0,1 , and $3 \times$ the synthesized beam. Using these imaging parameters, the combined continuum data at each band was iteratively selfcalibrated. The continuum peak position in all bands changed by $<0.8$ mas following self-calibration. The final synthesized beams and rms noise levels are given in Table 1 .

Even with ALMA's impressive collecting area, the surface brightness sensitivity at very high angular resolution is limited for detecting narrow thermal lines. To improve the surface brightness sensitivity, the data for the four Band 3 line transitions were tapered to a resolution of $\sim 1$." 1 (and later convolved to exactly this value) and imaged with robust $=0.5$ and a velocity width of $0.25 \mathrm{~km} \mathrm{~s}^{-1}$. Both the ${ }^{12} \mathrm{CO}(1-0)$ and $\mathrm{HCO}^{+}(1-0)$ lines show significant extended emission at this resolution and were cleaned with the same multi-scale parameters as the continuum (an additional scale of 45 was used for $\mathrm{CO})$. The $\mathrm{CN}(1-0)$ and $\mathrm{HCN}(1-0)$ lines are only detected in absorption toward the continuum peak. The resulting rms noise levels for the $1^{\prime \prime} 1$ resolution $\operatorname{HCN}(1-0)$, $\mathrm{HCO}^{+}(1-0), \mathrm{CN}(1-0)$, and ${ }^{12} \mathrm{CO}(1-0)$ cubes are 2.5, 2.5, 5.0, and $9.0 \mathrm{mJy}$ beam $^{-1}$, respectively (the $1^{\prime \prime} 1$ taper reduces the effective number of antennas to $\approx 20)$. Additionally, an $\mathrm{HCO}^{+}(1-0)$ line cube was made at a taper corresponding to an angular resolution of $\sim 0 . " 25\left(0 .{ }^{\prime \prime} 28 \times 0\right.$. ". 22 , P.A. $\left.=+40.5\right)$ with robust $=0.0$, and $0.25 \mathrm{~km} \mathrm{~s}^{-1}$ channels in order to explore the compact disk emission; the rms noise in this cube is $2.4 \mathrm{mJy} \mathrm{beam}^{-1}$. At this resolution ${ }^{12} \mathrm{CO}(1-0)$ still shows significant confusing contamination from outflow emission as well as copious self-absorption, while the $\mathrm{CN}(1-0)$ and $\mathrm{HCN}(1-0)$ lines are only marginally detected. We note that the continuum self-calibration was not applied to the line data, as tests demonstrated that this did not improve the S/N.

\section{REFERENCES}

ALMA Partnership, Fomalont, E. B., Vlahakis, C., et al. 2015, ApJL, 808, L1 (ALMA I)

Astropy Collaboration Robitaille, T. P., Tollerud, E. J., et al. 2013, A\&A, 558, AA33

Andrews, S. M., \& Williams, J. P. 2005, ApJ, 631, 1134

Anglada, G., López, R., Estalella, R., et al. 2007, AJ, 133, 2799

Baruteau, C., \& Papaloizou, J. C. B. 2013, ApJ, 778, 7

Beck, T. L., Bary, J. S., \& McGregor, P. J. 2010, ApJ, 722, 1360

Beckwith, S. V. W., \& Sargent, A. I. 1991, ApJ, 381, 250

Briceño, C., Luhman, K. L., Hartmann, L., Stauffer, J. R., \& Kirkpatrick, J. D. 2002, ApJ, 580, 317

Butler, R. P., Wright, J. T., Marcy, G. W., et al. 2006, ApJ, 646, 505

Cabrit, S., Guilloteau, S., Andre, P., et al. 1996, A\&A, 305, 527

Carrasco-González, C., Rodríguez, L. F., Anglada, G., \& Curiel, S. 2009, ApJL, 693, L86

Chiang, E. I., \& Goldreich, P. 1997, ApJ, 490, 368

Close, L. M., Roddier, F., Hora, J. L., et al. 1997, ApJ, 489, 210

Cornwell, T. J. 2008, ISTSP, 2, 793

Dias, W. S., Monteiro, H., Caetano, T. C., et al. 2014, A\&A, 564, AA79

Dong, R., Zhu, Z., \& Whitney, B. 2014, arXiv:1411.6063

Foreman-Mackey, D., Hogg, D. W., Lang, D., \& Goodman, J. 2013, PASP, 125,306

Forgan, D., Ivison, R. J., Sibthorpe, B., Greaves, J. S., \& Ibar, E. 2014, MNRAS, 439, 4057

Gómez, J. F., \& D’Alessio, P. 2000, ApJ, 535, 943

Goździewski, K., \& Migaszewski, C. 2014, MNRAS, 440, 3140

Greaves, J. S., Richards, A. M. S., Rice, W. K. M., \& Muxlow, T. W. B. 2008, MNRAS, 391, L74

Guilloteau, S., Dutrey, A., Piétu, V., \& Boehler, Y. 2011, A\&A, 529, AA105

Kenyon, S. J., \& Hartmann, L. 1995, ApJS, 101, 117

Kitamura, Y., Momose, M., Yokogawa, S., et al. 2002, ApJ, 581, 357

Krist, J. E., Stapelfeldt, K. R., Hester, J. J., et al. 2008, AJ, 136, 1980

Kwon, W., Looney, L. W., \& Mundy, L. G. 2011, ApJ, 741, 3

Lay, O. P., Carlstrom, J. E., \& Hills, R. E. 1997, ApJ, 489, 917

Lissauer, J. J., Ragozzine, D., Fabrycky, D. C., et al. 2011, ApJS, 197, 8

Loinard, L. 2013, in IAU Symp. 289, Advancing the Physics of Cosmic Distances (Cambridge: Cambridge Univ. Press), 36

Looney, L. W., Mundy, L. G., \& Welch, W. J. 2000, ApJ, 529, 477

Lumbreras, A. M., \& Zapata, L. A. 2014, AJ, 147, 72

Men'shchikov, A. B., Henning, T., \& Fischer, O. 1999, ApJ, 519, 257

Monin, J.-L., Pudritz, R. E., \& Lazareff, B. 1996, A\&A, 305, 572

Moriarty-Schieven, G. H., Johnstone, D., Bally, J., \& Jenness, T. 2006, ApJ, 645,357

Mundt, R., Buehrke, T., Solf, J., Ray, T. P., \& Raga, A. C. 1990, A\&A, 232,37

Mundy, L. G., Looney, L. W., Erickson, W., et al. 1996, ApJL, 464, L169

Nero, D., \& Bjorkman, J. E. 2009, ApJL, 702, L163

Pérez, L. M., Carpenter, J. M., Chandler, C. J., et al. 2012, ApJL, 760, LL17

Pyo, T.-S., Hayashi, M., Kobayashi, N., et al. 2006, ApJ, 649, 836

Rau, U., \& Cornwell, T. J. 2011, A\&A, 532, AA71

Rebull, L. M., Wolff, S. C., \& Strom, S. E. 2004, AJ, 127, 1029

Robitaille, T. P., Whitney, B. A., Indebetouw, R., \& Wood, K. 2007, ApJS, 169, 328

Rodmann, J., Henning, T., Chandler, C. J., Mundy, L. G., \& Wilner, D. J. 2006, A\&A, 446, 211

Roeser, S., Demleitner, M., \& Schilbach, E. 2010, AJ, 139, 2440

Sargent, A. I., \& Beckwith, S. V. W. 1991, ApJL, 382, L31

Schaefer, G. H., Dutrey, A., Guilloteau, S., Simon, M., \& White, R. J. 2009 , ApJ, 701, 698

Shen, Y., \& Turner, E. L. 2008, ApJ, 685, 553

Stapelfeldt, K. R., Burrows, C. J., Krist, J. E., et al. 1995, ApJ, 449, 888

Stephens, I. W., Looney, L. W., Kwon, W., et al. 2014, Natur, 514, 597

Takami, M., Beck, T. L., Pyo, T.-S., McGregor, P., \& Davis, C. 2007, ApJL, 670, L33

Welch, W. J., Hartmann, L., Helfer, T., \& Briceño, C. 2000, ApJ, 540, 362 White, R. J., \& Hillenbrand, L. A. 2004, ApJ, 616, 998

Wilner, D. J., Ho, P. T. P., \& Rodriguez, L. F. 1996, ApJL, 470, L117

Zacharias, N., Finch, C. T., Girard, T. M., et al. 2013, AJ, 145, 44

Zhang, X., Li, H., Li, S., \& Lin, D. N. C. 2014, ApJL, 789, LL23 